\title{
El psicólogo interno residente (PIR) en atención primaria: experiencia en el Complejo Hospitalario Universitario de Albacete
}

\author{
María Antònia Font Payeras \\ Hospital General de Villarrobledo (SESCAM)
}

María de la Merced Novo Vázquez

Unidad de Salud Mental de Jaca

Anabel Yanina Lemus Veleda

Complejo Hospitalario Universitario de Albacete

\section{RESUMEN}

En los últimos tiempos se ha detectado un interés creciente en la inclusión de psicólogos clínicos (PC) en el ámbito de la atención primaria $(A P)$, puesto que una parte significativa de las consultas de este nivel están relacionadas con problemas de salud mental, aumentando la presión asistencial de los médicos y ocasionando un elevado coste económico. La presencia del PC en AP mejoraría la accesibilidad de este servicio, revirtiendo en una mejor calidad asistencial. En varios países se han implementado programas en esta línea, existiendo algunos intentos pioneros en España. Uno de los cambios significativos en este sentido ha sido la inclusión desde el año 2009 de la rotación por AP como obligatoria en el programa de formación de los Psicólogos Internos Residentes (PIR). En este artículo se presenta la rotación realizada durante varios años en el Complejo Hospitalario Universitario de Albacete, así como su evolución y propuestas de mejora.

Palabras clave: psicología clínica; psicólogo interno residente (PIR); atención primaria; formación; sistema nacional de salud

\author{
María Dolores Gómez Castillo \\ Colegio Oficial de Psicólogos de Castilla la Mancha \\ Roger Muñoz-Navarro \\ Universitat de València
}

\begin{abstract}
In last years a growing interest has been detected with regards the inclusion of clinical psychologists $(C P)$ in Spanish primary care (PC), since significant amount of consultations at this level are related with mental health problems, increasing the pressure in medical care and causing a huge economic cost. The presence of the $C P$ in $P C$ would improve the access of this service, ending in a better quality of health care. Several countries have implemented programs in this line, as exist several attempts in Spain. One of the significant changes in this regard has been the inclusion since 2009 of the rotation in PC as compulsory in the training program internal resident psychologists (IRP). In this article, we present rotation performed for several years in the University Hospital of Albacete, as well as its evolution and some proposals for improvement.
\end{abstract}

Keywords: clinical psychology; internal resident psychologists; primary care; training; national health system 
La atención primaria (AP) es la puerta de entrada al sistema sanitario para las personas con problemas físicos, psicosociales o de salud mental. Tanto en España como en otros países, existe un amplio consenso en relación a la importancia de atender adecuadamente las problemáticas relacionadas con salud mental desde este nivel (Cano-Vindel, 2011; Layard, 2006). Distintos estudios (Caballero et. al., 1999; Roca et al., 2009) informan de que entre el 25 y el 33\% de las consultas de AP están relacionadas con problemas psicológicos, siendo los más frecuentes los cuadros depresivos con una prevalencia estimada del 5-9\% (Téllez, 2005). Estos problemas están directamente asociados a problemas psicosociales y estrés, lo que ha generado que en época de crisis, los problemas de salud mental hayan aumentado considerablemente. Concretamente, ha aumentado la presencia en las consultas de atención primaria de pacientes con trastorno depresivo mayor, distimia, trastorno de ansiedad generalizada, trastornos somatoformos y trastorno de pánico en un 19,4\%; 10,8\%; 8,4\%; $7,2 \%$ y un $6,4 \%$, respectivamente (Gili, Roca, Basu, McKee, \& Stuckler, 2013).

Siguiendo las recomendaciones de la Organización Mundial de la Salud (OMS), es necesario fomentar el aumento de profesionales con formación en los diferentes niveles de asistencia, incluido el de AP, que permita reducir las barreras que impidan que se preste la atención psicológica necesaria a estos pacientes (WHO \& WONCA, 2008). Chiles, Lambert y Hatch (1999), tras revisar 91 ensayos aleatorizados concluyen que una atención integrada entre médicos de atención primaria (MAP) y psicólogos clínicos (PC) puede reducir el coste sanitario total en torno a un $17 \%$. La atención en este primer nivel permitiría una mayor accesibilidad de los pacientes a la intervención psicológica, reduciendo tanto la sobrecarga en las consultas de los médicos de familia como la lista de espera en atención especializada (AE) de Salud Mental (SM), al derivar sólo aquellos casos de mayor gravedad. Una buena parte de las demandas de AP se centran en problemas de afrontamiento y adaptación a diversas enfermedades físicas (Friedman, Sobel, Myers, Caudill, \& Benson, 1995) y a otras situaciones estresantes, así como problemas emocionales leves. Este tipo de problemática puede ser abordada por los PC de AP mediante intervenciones psicológicas eficaces de baja intensidad, tales como terapias de resolución de problemas (García-Campayo, Hidalgo, \& Orozco, 2006) o activación conductual (Dimidjian et al., 2006). De este modo, se logran reducir las derivaciones $A E$, y la consiguiente estigmatización.

En esta línea, diversos países europeos han llevado a cabo programas de mejora de acceso a tratamientos psicológicos desde la atención primaria. Dos ejemplos de modelos de intervención psicológica en AP se han desarrollado en el Reino Unido y Noruega. El primero de ellos instaura, con la reforma sanitaria de 2007, un modelo de atención psicológica paso a paso denominado Improving Acces to Psicological Therapies (IAPT) dirigido a población adulta con trastorno mental común, principalmente cuadros de depresión y ansiedad (Turpin, Richards, Hope, \& Duffy, 2008), proporcionando una intervención escalonada en función de la gravedad de los trastornos, existiendo cuatro 
niveles de atención creciente. Este proyecto plantea la dispensación de una terapia de corte cognitivo-conductual como tratamiento de primera elección, dada la mayor evidencia existente sobre su efectividad en el tratamiento de estos trastornos, si bien, no se excluye la posibilidad de utilizar algún otro enfoque. El National Institute for Health and Care Excellence (NICE) (2004), organismo asesor del ejecutivo británico en materia de salud, recomienda este modelo de intervención, al considerar que la terapia psicológica es más eficaz que la medicación y que debería estar disponible para todos los pacientes que la necesiten.

En el caso de Noruega, el modelo de tratamiento psicológico actual se introdujo con el Plan de Salud Mental iniciado en el Parlamento en 1998, tratándose de un enfoque basado en la perspectiva del usuario y que favorece por tanto su autonomía, apostando por facilitar el acceso al tratamiento psicológico desde intervenciones de baja intensidad, al igual que en el caso del Reino Unido. De este modo ha pasado de ofrecer tratamiento ambulatorio especializado para personas con trastornos mentales graves, a proporcionar intervenciones psicológicas tempranas desde AP para problemas mentales leves y moderados. Estas pueden ser tanto individuales como grupales, sin que existan criterios de exclusión en función de la edad. Este cambio ha permitido aumentar la prevención e intervención temprana, incrementando también la coordinación y cooperación entre los profesionales involucrados, al tiempo que disminuye tanto la duración de la psicoterapia (media de cinco consultas, con un rango de entre una y quince) como las deriva- ciones a $A E$, acudiendo a ese nivel el 5-10\% de los casos (Johnson, 2012).

\section{La Psicología Clínica en la atención primaria española}

En los últimos años, por tanto, se observa una creciente inquietud por intentar implementar programas semejantes a la realidad española. Asimismo, el Consejo General de Colegios Oficiales de Psicólogos (CGCOP) denuncia el difícil acceso de los pacientes a una terapia psicológica en el ámbito de la sanidad pública, ya que se trata de un servicio que en el Sistema Nacional de Salud (SNS) español se localiza en el nivel de AE y no en AP, siendo el componente psicológico en estas consultas, tal y como se ha indicado anteriormente, muy elevado (Gómez, s.f.). Además, estos trastornos están excesivamente medicalizados en este contexto. Moreno y Moriana (2012) señalan que la excesiva medicalización de problemas leves puede resultar iatrogénica, dificultando el desarrollo de habilidades de afrontamiento adecuadas, así como aumentando el riesgo de cronificación, con el elevado coste económico que esto supone. En este sentido, Sicras et al. (2007) refieren que el gasto que suponen los casos de depresión es un $58,4 \%$ superior al de enfermedades físicas crónicas, debiéndose el 64\% del mismo exclusivamente a los fármacos. Por tanto, la inclusión de $P C$ en $A P$, según se recomienda a nivel mundial (WHO \& WONCA, 2008) permitiría paliar las limitaciones existentes, mejorando la eficiencia y la calidad asistencial.

En España, la primera experiencia de integración del PC en AP se llevó a cabo en Murcia. Según se detalla en una publicación del Cole- 
gio Oficial de Psicólogos de la Región de Murcia ("Primera experiencia en Murcia", s.f.) se inició en el año 2011, a raíz del terremoto de Lorca, con la contratación de dos psicólogos clínicos. Se desarrolló un protocolo de evaluación y, posteriormente, a partir de la intervención escalonada según la gravedad de cada caso, se diseñaron 4 grandes grupos: 1) normalización de casos de reacción a estrés agudo (una sesión y seguimiento); 2) intervención grupal (cuatro o cinco sesiones, con seguimiento a los tres meses); 3) intervención individual, con un máximo de 9 sesiones; 4) derivación a salud mental, en caso de trastorno mental grave o patología previa. A raíz de la valoración positiva de esta experiencia decidieron mantener un modelo de atención escalonada y ese mismo año, introducirlo en al área de Cartagena, con intención de ir extendiéndolo progresivamente.

Otra experiencia de integración en España es el Programa de Soporte a Atención Primaria (PSP) en la provincia de Barcelona. Los objetivos principales son promover la integración de la Salud Mental en la actividad de los equipos de AP, fomentar un cambio cultural de derivar a compartir, potenciar los recursos disponibles para la contención de síntomas derivados del malestar de la vida cotidiana y despatologizar, mejorar la capacidad de detección e intervención desde AP en lo referente a salud mental, y dar continuidad asistencial a través del trabajo en red. Se trata de un planteamiento que facilita la existencia de un espacio de interconsulta entre el MAP y el psicólogo clínico en aspectos como la detección, orientación o decisión de tratamiento. Además, se incide en identificar factores de riesgo y señales de alarma, propor- cionar información de los recursos asistenciales, hacer devolución de la orientación diagnóstica, los objetivos terapéuticos y la evolución, así como realizar la derivación a Salud Mental en casos de trastorno mental grave. En este programa se tratan aquellos problemas leves y situaciones vitales que requieren atención psicológica, además de los trastornos de gravedad moderada, con repercusión funcional desadaptativa, siempre que sean abordables desde este nivel.

A pesar de estos proyectos, es necesario seguir abordando la necesaria implementación de psicólogo clínico en atención primaria de forma más extensa. Tal y como muestra el Real Decreto 1030/2006, que regula la cartera de servicios del Sistema Nacional de Salud, es importante contemplar el abordaje de la salud mental en AP, tanto la vertiente de detección de psicopatología como el tratamiento de trastornos adaptativos, depresión y ansiedad. Por otro lado, la Estrategia en Salud Mental del Sistema Nacional de Salud 2009-2013 (Ministerio de Sanidad, 2011) establece como objetivo específico «aumentar el porcentaje de pacientes con trastornos psicológicos que reciben psicoterapia, acorde con las mejores prácticas disponibles y monitorizando criterios mínimos de calidad contemplados». Las notas de prensa relativas a la última actualización de esta Estrategia (2015-2020, pendiente de publicación) transmiten una continuidad en esta línea, manteniendo el énfasis en la necesidad de abordar la problemática a la que se enfrentan las personas con trastornos psicológicos, existiendo un grupo de trabajo específico en relación con la coordinación entre AP y SM. 
Recientemente, se ha alcanzado la aprobación de dos Proposiciones No de Ley (PNL) apostando por la creación de la figura del PC en AP, una en la Asamblea de Madrid (BOAM, 2016) y otra en la Comunidad Valenciana $(B O C V, 2016)$. Estos avances sugieren que pronto se podrán ir dando pasos en la consumación de estos objetivos. Para ello, es necesario que los profesionales que dispensen las terapias psicológicas en el Sistema Nacional de Salud y en este caso, en la AP cuenten con la mejor formación posible para asegurar la mejor atención de estos pacientes y su recuperación.

Finalmente, otra de las experiencias relevantes que giran en torno a este objetivo en nuestro país es el ensayo PsicAP-Psicología en Atención Primaria. Se trata de un proyecto impulsado por Psicofundación, (Fundación Española para la Promoción y el Desarrollo Científico y Profesional de la Psicología en el Ámbito Español), promovida por el Consejo General de Colegios Oficiales de Psicólogos para impulsar de forma activa todas las acciones necesarias encaminadas a la participación y adaptación del colectivo profesional de psicólogos en la consecución de estos retos. En esta línea, iniciaron en el año 2010 acciones encaminadas para llevar a cabo un ensayo clínico en el sistema sanitario público para demostrar la eficacia de los tratamientos psicológicos basados en la evidencia para los desórdenes emocionales en AP (Cano-Vindel et al., 2016). Por último, tras la aprobación de los comités éticos, se inició en diciembre del 2013 un ensayo clínico aleatorizado que está implantado actualmente en 22 centros de AP de más de 7 comunidades autónomas del territorio español. Este ensayo tiene dos grandes objetivos, poner a prueba un tratamiento grupal de corte transdiagnóstico para los desórdenes emocionales frente al tratamiento convencional llevado a cabo en atención primaria (tratamiento farmacológico por los MAP) y, además, realizar una serie de medidas de costo-eficacia con el gran objetivo de demostrar al Ministerio de Sanidad, así como de las Consejerías de Sanidad autonómicas, los beneficios para la salud de la población y la mejora de la sostenibilidad del sistema sanitario público español, así como la economía en su conjunto, a partir de la incorporación del PC en AP.

\section{Objetivos}

En esta línea, los proyectos internacionales mencionados previamente sirven como ejemplo a seguir $y$, con el fin de adaptar estos a la realidad española donde se cuenta con el modelo de formación PIR, se exponen dos objetivos en el presente trabajo. El primero es mostrar la experiencia de la rotación PIR en la Comunidad de Castilla La Mancha, en concreto en el Complejo Hospitalario de Albacete, donde esta rotación se lleva realizando desde el año 2011. Finalmente, como segundo objetivo, se proponen una serie de mejoras de la rotación PIR a partir de nuestra experiencia para que los futuros psicólogos clínicos cuenten con la mejor preparación posible para afrontar los grandes retos que supone la inserción de la figura del PC en AP, siguiendo algunas de las propuestas del ensayo PsicAP. 


\section{La rotación del psicólogo interno residente} en atención primaria.

La experiencia en Albacete.

El Complejo Hospitalario Universitario de Albacete lleva años formando residentes de psicología clínica, existiendo actualmente un residente por año. El Programa Oficial de Formación de Psicólogos Especialistas en Psicología Clínica sufrió un proceso de modificación en 2009, tal como consta en el Boletín Oficial del Estado, consistiendo el principal cambio en ampliar el periodo de residencia a 4 años. Esta modificación incluía nuevas rotaciones, destacando la rotación obligatoria por AP, que en este hospital se introdujo en el año 2011.

\section{Objetivos de la rotación}

Los objetivos generales que se plantearon al inicio de la rotación en AP fueron:

- Mejorar el reconocimiento de las necesidades asistenciales de salud mental en dispositivos médicos.

- Conocer los aspectos básicos de la organización, funcionamiento y gestión de los Centros de AP.

- Participar en el funcionamiento de los equipos multidisciplinares y espacios de coordinación de AP.

- Aportar un rápido feedback de las apreciaciones al profesional médico.

- Conocer los criterios de derivación a atención especializada, sobre todo a los dispositivos de Salud Mental.

- Profundizar en las psicopatologías más prevalentes en este nivel y su diagnóstico diferencial, así como en los trastornos somáticos asociados.
- Valorar el impacto de la enfermedad física sobre el estado mental y la calidad de vida del paciente y de su familia.

- Adquirir conocimientos sobre el seguimiento de los trastornos crónicos desde Atención Primaria.

- Conocer y participar en programas de promoción de la salud y prevención de la enfermedad, entendido desde un modelo biopsicosocial.

\section{Temporalidad de la rotación}

La rotación se realizó en un único centro de salud de Albacete, que atiende a población de ámbito urbano. El inicio de la rotación se efectuó durante un mes en la consulta del MAP, reduciéndose progresivamente este período en las últimas rotaciones. Desde el principio, se consideró importante la continuidad de esta atención psicológica en AP, por lo que cada residente permanece un día a la semana durante un año, de manera que cuando finaliza su rotación, inmediatamente después enlaza un nuevo residente.

\section{Sistema de derivación de pacientes a los residentes}

El modo de derivación consiste en que los MAP del Centro de Salud donde se realiza la rotación deriven al residente aquellos pacientes susceptibles de tratamiento psicológico. Éste asume los casos que se puedan beneficiar de un programa de intervención breve, que serían aquellos que presentan trastornos o reacciones adaptativas, así como trastornos psicológicos leves/moderados de corta evolución y que no generen incapacidad grave. Por 
otro lado, se derivan a $\mathrm{AE}$ aquellos con patología crónica o de mayor gravedad y que por ello necesitarían un tratamiento más continuado. (Fig. 1).

\section{Intervenciones y tratamientos ofrecidos}

Con este modo de trabajo se pretende proporcionar una atención temprana a través de intervenciones grupales protocolizadas y tratamientos psicológicos individuales. En ambos casos se trata de intervenciones breves, con una duración entre tres y seis meses, intensivas puesto que existe la posibilidad de citación semanal, y con acceso rápido, siendo atendidos en la primera semana tras la derivación.

A modo de ejemplo, a partir de una de las rotaciones se elaboró una base de datos de la que se extrajo información relativa al perfil de los pacientes atendidos en ese período. Prácticamente en la totalidad de los casos la demanda partía del MAP, debido a que la posibilidad de recibir atención psicológica en el centro de salud no era conocida por la población. La muestra estaba formada por 19 pacientes, de cuales 7 eran varones y 12 mujeres. El rango de edad era de 20 años a 85 años, con una media de 49 años, siendo el grueso mujeres de mediana edad. Un 63\% recibía tratamiento farmacológico pautado por su MAP. Los diagnósticos más prevalentes eran los cuadros adaptativos, con un $37 \%$, seguidos de trastornos depresivos y trastornos de ansiedad. En el momento de la derivación el tiempo de evolución variaba desde cuadros de reciente aparición, a otros de larga evolución. Se procedió al alta por mejoría al $42 \%$ de los pacientes tras una media de ocho sesiones de tratamiento, siendo éstos cuadros adaptativos y trastornos de ansiedad. Del resto de casos, sólo fue necesario derivar a atención especializada a un $15 \%$ del total.

Por otro lado, otra experiencia de la que constan datos es una intervención grupal cog-

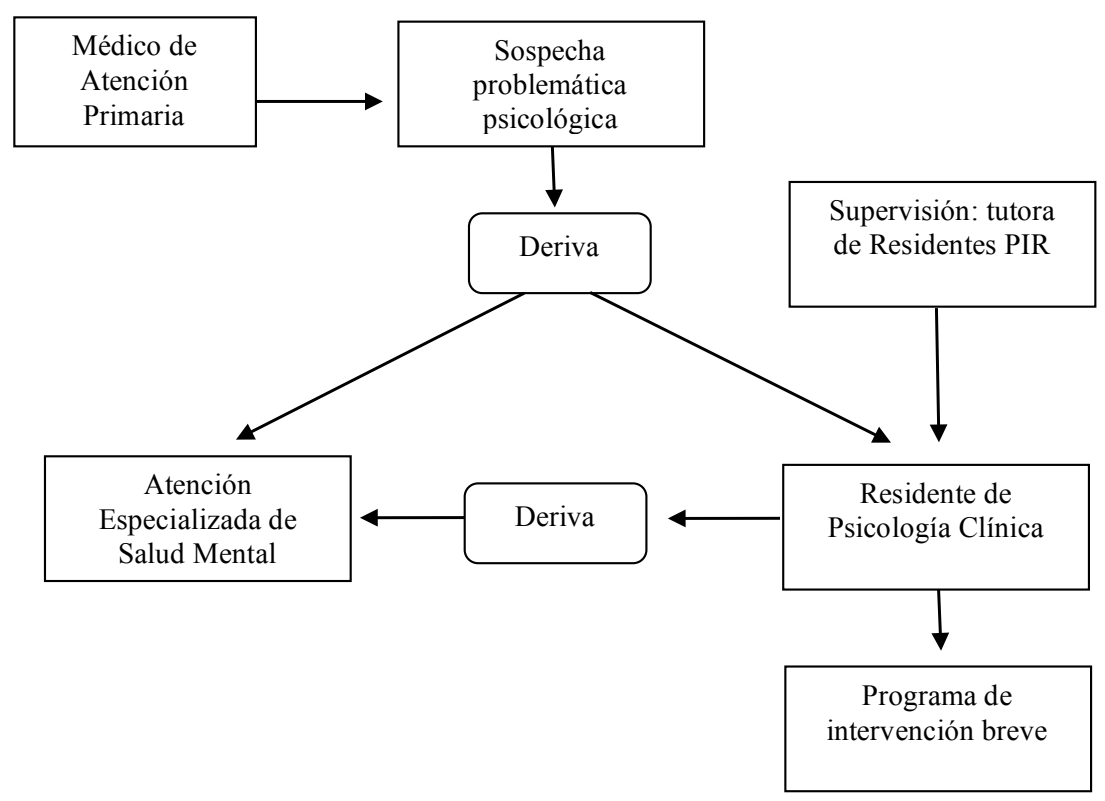

Figura 1. Protocolo de derivación. 
nitivo conductual. Los criterios de inclusión eran adultos de 18 a 65 años, con diagnóstico principal de trastorno de ansiedad, preferencia por el abordaje psicológico y motivación hacia el tratamiento grupal, con cierto nivel de conciencia para poder beneficiarse del tratamiento. Los criterios de exclusión establecidos eran presentar discapacidad intelectual y/o presencia de depresión grave, trastorno mental grave, conductas auto y/o heteroagresivas o trastornos relacionados con sustancias. El grupo estaba formado por seis pacientes (cuatro mujeres y dos varones) con edades comprendidas entre los 20 y los 49 años. Se realizaron diez sesiones de 90 minutos de duración, con una frecuencia semanal. Se realizó una evaluación inicial y postratamiento mediante el Índice de Sensibilidad a la Ansiedad (ASI, Anxiety Sensitivity Index; Peterson y Reiss, 1992; Sandín et al., 2004), el Inventario de Depresión de Beck- II (BDI-II, Beck Depression Inventory; Beck et al., 1996; Beck, Steer, \& Brown, 2011) y el Inventario de Ansiedad de Beck (BAl, Beck Anxiety Inventory; Beck et al.,1988; Beck \& Steer, 2011). Se obtuvo una mejoría general en las puntuaciones medias de todos los cuestionarios. Destaca el cambio observado en la puntuación media del BAI con una diferencia de 14 puntos, siendo la puntuación media inicial de 33 y la final de 19, lo que implica pasar de una ansiedad moderada a baja. Estos resultados, que no se exponen como datos empíricos sino como una muestra descriptiva de la actividad de la rotación en Albacete, reflejan desde la práctica clínica lo que la evidencia científica muestra, que los tratamientos psicológicos en AP son eficaces para estos problemas.

\section{Dificultades encontradas}

Se han encontrado obstáculos en la puesta en marcha de distintos objetivos iniciales. $A$ causa de la inexistencia de la figura de PC en este ámbito, y además, el hecho de que la rotación se realizara un sólo día a la semana y en horario de tarde, se ve limitada la capacidad de coordinación y trabajo en equipo con los distintos profesionales. Por otra parte, hay que considerar que ha existido una ausencia de incorporación de residentes en algunas convocatorias previas, lo que se venía solventando hasta la actualidad adelantado ésta a años tempranos de la residencia. Otra alternativa para paliar este inconveniente sería mediante la discontinuidad de un año de la rotación, lo cual supondría la interrupción de la actividad asistencial desarrollada. A lo largo de estos años, la atención se ha limitado a la población adulta, mientras que en la población infantil no se han realizado ninguna intervención. Además, otro aspecto que hasta ahora no se ha podido desarrollar de manera óptima ha sido la prevención y promoción de la salud, realizándose de forma puntual, sin la existencia de programas establecidos.

\section{Propuestas de mejora futuras de la rotación PIR en AP}

Como segundo objetivo de este trabajo, y tras la exposición de la experiencia de rotación PIR en AP a partir de la Residencia del Complejo Hospitalario de Albacete, se pretende hacer una serie de propuestas de mejora para dicha rotación que se consideran que podrían resultar beneficiosas. En esta línea, se propone no sólo contar con la experiencia de los propios 
residentes si no también realizar un aporte desde la investigación, concretamente, desde el ensayo PsicAP. Ambas perspectivas unificadas, pueden ser de gran valor para contemplar posibles cambios en la rotación PIR.

\section{Objetivos de la rotación}

Frente a todos los objetivos mostrados anteriormente, que ya están contemplados en la actualidad, se observa que el sistema de rotación actual dificulta la consecución de algunos de ellos. Además, como se ha mencionado, hay dos objetivos que deberían ser primordiales.

\section{Mejora del sistema de detección y derivación}

El primer objetivo se enmarcaría en una mejora del sistema de detección, diagnóstico y tratamiento de aquellos problemas de salud mental y física que deberían recibir intervención psicológica desde la propia AP (terapias de baja intensidad). Y, además de la derivación de aquellos pacientes que requerirían una intervención en servicios especializados y hospitalarios (terapias de alta intensidad), en coordinación con los MAP y otros profesionales. Este objetivo se propondrá en un aparatado posterior.

\section{Atención a la población infantil-juvenil}

Por otra parte, sería deseable que uno de los objetivos fuera que el PC en AP incluyera la intervención sobre la población infantil y juvenil. Por tanto, sería necesario dedicar un tiempo de trabajo en coordinación con el servicio de pediatría, para poder abordar todos los aspectos que requieren intervención psicológica desde la propia AP y/o derivación a otros servicios. Actualmente, en Albacete, se está iniciando la colaboración con una pediatra de AP, para así ampliar el trabajo asistencial a esta población y realizar intervenciones relacionadas con distintas patologías en función de la demanda, ya sea por su prevalencia o dificultad de manejo.

\section{Temporalidad de la rotación}

La temporalidad de la rotación es un aspecto muy importante para poder alcanzar estos objetivos. De hecho, esta rotación debería ser en el último año de residencia, ya que estos ya tendrían una visión mucho mayor del funcionamiento de todo el sistema sanitario público español y de todas las patologías, lo que ayudaría a identificar a los pacientes y derivarlos al servicio más adecuado.

\section{Nuevo sistema de derivación de pacientes a los residentes}

A raíz de los estudios que se están realizando en el ensayo PsicAP, se cuenta con pruebas psicométricas validadas que pueden ayudar a reducir el infradiagnóstico de los problemas de salud mental más importantes, la ansiedad y la depresión. Concretamente, se ha observado que el uso de pruebas como el PHQ-9, el GAD-7 y el PHQ-PD resulta útil para detectar los casos de depresión (Muñoz-Navarro et al., 2017b), trastorno de ansiedad generalizada (MuñozNavarro et al., 2017b) y pánico, respectivamente (Muñoz-Navarro et al., 2016). Además, el uso de pruebas ultracortas, como el PHQ-4 que cuenta con 2 ítems para detectar depresión (PHQ2) y 2 para el GAD (GAD-2) han mostrado una alta sensibilidad con puntos de corte de 3 para 
cada prueba $(0,90$ y 0,88 , respectivamente) (Muñoz-Navarro et al., 2017c). Además, tan solo la pregunta de cribado del PHQ-TP (En las últimas 4 semanas ¿ha tenido algún ataque de ansiedad - sensación repentina de miedo o pánico?), ha mostrado una alta sensibilidad $(0,83)$ (Muñoz-Navarro et al., 2016). Estas pruebas son muy sensibles, lo que permitiría reducir el infradiagnóstico que ocurren en la AP española (Fernández et al., 2010) pero desgraciadamente carecen de especificidad, por lo que pueden hallar muchos falsos negativos.

Aquí es donde los residentes en AP podrían llevar a cabo un trabajo de evaluación y diagnóstico con entrevistas clínicas estructuradas, que permitirían afinar la especificidad $y$, por tanto, derivar al servicio especializado del SNS en los casos necesarios (terapias de alta intensidad) y tratar lo adecuado en AP (terapias de baja intensidad y prevención y promoción de la salud).

Por tanto, el modelo de derivación que se propone para la formación de los futuros residentes, conlleva también la formación de los MAP y de otros profesionales de los centros de AP, en el uso de estas pruebas de cribado, con el objetivo de que llegaran a psicólogos que realizaran sus residencias en AP. Este procedimiento facilitaría la detección de casos de personas con problemas de salud mental, que se sabe que hoy en día, son los problemas de mayor prevalencia y que están generando pérdidas de cerca del 4,5\% del Producto Interior Bruto (PIB) español, según recientes estudios (Parés-Badell et al., 2014).

En este planteamiento teórico y poco viable en la actualidad, se basaría en un proceso es- calonado y colaborativo entre los MAP y los residentes en un modelo jerárquico de diagnóstico y derivación de los trastornos mentales AP (Ver Muñoz-Navarro et al., 2017c para más detalle). En este modelo, se propone el uso del PHQ-4, más la pregunta de cribado del PHQTP (5 ítems en total) que constituiría el primer escalón. En un segundo escalón, se contaría con otras pruebas más amplias, como el PHQ con sus módulos, el PHQ-9, GAD-7, PHQ-PD, PHQ-15, para detectar, depresión, GAD, pánico y somatizaciones, respectivamente. Una vez llegaran estas puntuaciones al residente, este realizaría un tercer escalón, con entrevistas clínicas diagnósticas estructuradas, tales como la SCID o la CIDI. Así, realizarían un diagnóstico confirmatorio y mejoraría la derivación a los servicios correspondientes. Por tanto, se formaría a los futuros residentes y PC que trabajaran en AP, a realizar el triaje de los problemas de salud mental en todo su continuo. Por eso, es tan importante que el psicólogo que trabaje en AP sea un PC en formación por la vía PIR, ya que es la mejor para trabajar en AP.

Cabe mencionar, que todas estas propuestas se engloban en un marco ideal al que cabría aspirar en un futuro en el que hubiera una inserción de PC en AP adecuado y ajustado a la demanda existente. Por tanto, deben ser consideradas como objetivos deseables, que se verían favorecidos en caso de que hubiera una mayor conciencia de las administraciones públicas por realizar la inserción del PC en AP, más allá o no de que sean más o menos alcanzables en un futuro próximo. 


\section{Intervenciones y tratamientos a ofrecer}

En AP, es conveniente que los $\mathrm{PC}$ y futuros residentes, conozcan el modelo escalonado de tratamiento, que cuenta con 5 escalones. Este modelo trata en un primer escalón aquellas cuestiones subclínicas con relajación y psicoeducación. En un segundo escalón, es importante que se lleven a cabo terapias de grupo de corte cognitivo conductual y transdiagnóstico, lo que facilitaría la creación de grupos mixtos, agilizando el servicio de terapias al alcance en poco tiempo y con mucha efectividad. En un tercer escalón, serían aquellas terapias individualizadas de baja intensidad, con un posible acompañamiento farmacológico dispensado por el MAP. Finalmente, el cuarto escalón sería en Unidades de Salud Mental, con atención ambulatoria, y el quinto en hospitales y centros de larga estancia.

\section{Conclusiones}

La AP es la vía de acceso de la población general al abordaje de problemas de diversa índole. En este sentido, algunos autores señalan la alta prevalencia de problemas psicológicos y los altos costes relacionados, la excesiva medicalización de los mismos y la necesidad de emplear tratamientos psicológicos como primera opción, sobre todo para los trastornos psicológicos comunes (Pastor-Sirera, 2008; Pérez-Álvarez, \& Fernández-Hermida, 2008). En este espacio podría ser relevante el papel del PC, figura que ya se está empezando a incluir en AP en diferentes países, y en diversas comunidades autónomas de nuestro país.

En cuanto a esta figura, en los últimos años se desprenden interesantes conclusiones en jornadas dedicadas a la Salud Mental en AP. A pesar de que el PC puede generar un apoyo relevante en este ámbito, no se encuentra en la actualidad una estructura estable que permita este tipo de intervención, que sería de gran interés para la población y el SNS. Se han propuesto mejoras en la línea de ofrecer una alternativa o un complemento al tratamiento psicofarmacológico y, así, despatologizar y desmedicalizar el abordaje; del mismo modo, plantear programas de prevención y rehabilitación, que sirvan como complemento a programas dirigidos a la educación para la salud. Asimismo, se ha planteado que esta inclusión en AP permitiría al paciente mayor acceso y continuidad en la atención psicológica, ampliando la oferta de servicios y disminuyendo el tiempo de espera para dicha atención (Consejo General de la Psicología España, 2013).

Para finalizar y siguiendo en la línea de consideraciones realizadas por otros autores, se podrían definir las siguientes líneas de actuación para el futuro: especificar de forma más clara los ámbitos y los papeles de la Salud Mental y la Psicología Clínica; facilitar la inclusión del PC en la AP y, por último, mejorar la producción psicológica en publicaciones relativas a la salud (Costa \& López, 2011).

Se podría considerar que estos puntos redundarían en múltiples ventajas, entre las que caben destacar la mejora de la calidad asistencial, la reducción de las listas de espera, la facilitación del acceso a la atención y la reducción de la estigmatización, así como la derivación solamente en casos necesarios a Atención Especializada. 
En cuanto a la experiencia particular de los PIR en el Complejo Hospitalario Universitario de Albacete, a pesar de las dificultades con las que nos hemos ido encontrando a la hora de instaurar esta rotación, se han conseguido mejoras de manera progresiva que están permitiendo un mayor ajuste de la misma a los objetivos planteados. Cabe destacar en este sentido la flexibilidad y capacidad de adaptación sucesiva en función de las circunstancias cambiantes. Por este motivo se han realizado una serie de propuestas que consideramos que pueden ser muy satisfactorias en la mejora de la formación de los futuros residentes de psicología clínica, las cuales están razonadas y justificadas a partir de la experiencia clínica y la investigación. Ambas, unidas de la mano podrán fomentar la creación de caminos que lleven a abordar uno de los grandes retos del futuro cada vez más cercano, la atención psicológica basada en la evidencia en la atención primaria.

\section{Referencias}

Beck, A. T., Steer, R. A., \& Brown, G. K. (1996). BDI-Il. Beck Depression Inventory manual. San Antonio, TX: The Psychological Corporation.

Beck, A. T., Epstein, N., Brown, G., \& Steer, R.A. (1988). An inventory for measuring clinical anxiety: Psychometric properties. Journal of Consulting and Clinical Psychology, 56, 893897.

Beck, A.T., \& Steer, R. A. (2011). Manual. BAI. Inventario de Ansiedad de Beck (Adaptación española: Sanz, J.). Madrid: Pearson Educación.

Beck, A. T., Steer, R. A., y Brown, G. K. (2011). Manual. BDI-Il. Inventario de Depresión de BeckII (Adaptación española: Sanz, J ., \& Vázquez, C.). Madrid: Pearson.
BOAM (2016). PNL 135/16 RGEP 6302. Recuperado de http://www.asambleamadrid.es/ FicherosAgenda/DOC1026377.pdf

BOCV (2016). Resolución 93/IX de fecha 06.07.2016. Recuperado de http://www. cortsvalencianes.es/BASISCGI/BASIS/BOCV/ WEB/BOCV_INS_C/DDW?W=CLAVE_INSER$\mathrm{CION}=188653297480782$.

Boletín Oficial del Estado (17 de Junio, 2009). Orden SAS/1620/2009, de 2 de junio, por la que se aprueba y publica el programa formativo de la especialidad de Psicología Clínica. Recuperado de https://www.boe.es/boe/ dias/2009/06/17/pdfs/BOE-A-2009-10107. pdf

Caballero, M.L., Fernández-Liria, A., Ramos, J.A., Gil, A., Madrigal, M., Porras, A., Baca, B.E.,... Agüera, L.F. (1999). Prevalencia de los trastornos psiquiátricos en atención primaria usando el cuestionario PRIME-MD. Atención Primaria, 23, 275-279.

Cano-Vindel, A., Muñoz-Navarro, R., Wood, C. M., Limonero, J. T., Medrano, L. A., Ruiz-Rodríguez, P., ... \& Santolaya, F. (2016). Transdiagnostic Cognitive Behavioral Therapy Versus Treatment as Usual in Adult Patients with Emotional Disorders in the Primary Care Setting (PsicAP Study): Protocol for a Randomized Controlled Trial. JMIR research protocols, 5(4).

Chiles, J. A., Lambert, M. J., \& Hatch, A. L. (1999). The Impact of Psychological Interventions on Medical Cost Offset: A Meta-analytic Review. Clinical Psychology: Science and Practice, 6, 204-220.

Consejería de Salud Mental de Castilla la Mancha (2015). Estrategia de Salud Mental de Castilla la Mancha 2015-2017. Recuperado de http://www.castillalamancha.es/sites/ default/files/documentos/pdf/20150629/ estrategia_salud_mental_2015-2017.pdf

Consejo General de la Psicología España, (11 de noviembre, 2013). Conclusiones: Jornada de Trabajo sobre Psicología Clínica en Atención Primaria. Recuperado de http://www. cop.es/uploads/PDF/JAP11-11-13.pdf

Costa, M ., \& López, E. (13 de Junio, 2011). Es necesario facilitar la presencia del psicólogo en los equipos de atención primaria. Info- 
cop. Recuperado de http://www.infocop.es/ view article.asp?id=3501

Dimidjian, S., Hollon, S. D., Dobson, K. S., Schmaling, K. B., Kohlenberg, R. J., Addis, M. E., Gallop, R.,... Jacobson, N. S. (2006). Randomized trial of Behaviorial Activation, Cognitive Therapy, and antidepressant medication in the Acute Treatment of Adult. Journal of Consulting and Clinical Psychology, 74, 658670.

Fernández, A., Pinto-Meza, A., Bellón, J. A., Roura-Poch, P., Haro, J. M., Autonell, J., ... \& Luciano, J. V. (2010). Is major depression adequately diagnosed and treated by general practitioners? Results from an epidemiological study. General hospital psychiatry, 32, 201-209.

Friedman, R., Sobel, D., Myers, P. Caudill, M., \& Benson, H. (1995). Behavioral Medicine, Clinical Health Psychology, and cost offset. Health Psychology, 14, 509-518.

García-Campayo J., Hidalgo, I., \& Orozco, F. (2006). Psicoterapia de resolución de problemas en atención primaria. Barcelona: Grupo Ars XXI de Comunicación.

Gili, M., Roca, M., Basu, S., McKee, M., \& Stuckler, D. (2013). The mental healthrisks of economic crisis in Spain: Evidence from primary care centres, 2006 and 2010. The European Journal of Public Health, 23, 103-108.

Gómez, M. D. (s.f.). Guía formativa de residentes de psicología clínica. Recuperado de http:// www.chospab.es/docencia/guias formativas/Psicologia.pdf

Johnson, R. (2012). Experience and research in psychological intervention in primary health care in Europe. En M. D. Gómez (Presidencia). Presentado en Symposium in Clinical Psychology in Primary Health Care. Madrid, España.

Layard, R. (2006). The case for psychological treatment centres. Bmj, 332 (7548), 10301302.

Ministerio de Sanidad, Política Social e Igualdad (2011). Estrategia en Salud Mental del Sistema Nacional de Salud 2009-2013. Recuperado de www.msssi.gob.es/organizacion/sns/planCalidadSNS/docs/saludmental/ SaludMental2009-2013.pdf
Moreno, E., \& Moriana, J.A. (2012). El tratamiento de problemas psicológicos y de salud mental en atención primaria. Salud Mental, 35, 315-328.

Muñoz-Navarro, R., Cano-Vindel, A., Medrano, L. A., Schmitz, F., Ruiz-Rodríguez, P., AbeIlán-Maeso, C., . . . Hermosilla-Pasamar, A. M. (2017a). Utility of the PHQ-9 to identify major depressive disorder in adult patients in Spanish primary care centres. BMC Psychiatry, 17(1), 291.

Muñoz-Navarro, R., Cano-Vindel, A., Moriana, J. A., Medrano, L. A., Ruíz-Rodríguez, P., Agüero, L., .. Ramírez-Manent, I. (2017b). Screening for generalized anxiety disorder in Spanish primary care centers with the GAD-7. Psychiatry Research,

Muñoz-Navarro, R., Cano-Vindel, A., Ruiz-Rodríguez, P., Medrano, L. A., González-Blanch, C., Moriana, J. A., ... \& Dongil-Collado, E. (2017c). Modelo jerárquico de diagnóstico y derivación de los trastornos mentales comunes en centros de atención primaria. Una propuesta a partir del ensayo clínico PsicAP. Ansiedad y Estrés.

Muñoz-Navarro, R., Cano-Vindel, A., Wood, C. M., Ruíz-Rodríguez, P., Medrano, L. A., Limonero, J. T., . . Iruarrizaga, M. I. (2016). The PHQ-PD as a screening tool for panic disorder in the primary care setting in Spain. PloS One, 11(8), e0161145.

National Institute for Health and Care Excellence (NICE) (2004). Depression: management of depression in primary and secondarycare: National Clinical Practice Guideline number 23. London: National Institute for Clinical Excellence.

Parés-Badell, O., Barbaglia, G., Jerinic, P., Gustavsson, A., Salvador-Carulla, L., \& Alonso, J. (2014). Cost of Disorders of the Brain in Spain. PloS One, 9(8), e105471.

Pastor Sirera, J. (2008). El psicólogo en atención primaria: un debate necesario en el sistema nacional de salud. Papeles del Psicólogo, 29(3), 271-290.

Pérez Álvarez, M., \& Fernández Hermida, J. R. (2008). Más allá de la salud mental: la psicología en atención primaria. Papeles del Psicólogo, 29(3), 251-270. 
Peterson, R.A., \& Reiss, R.J. (1992). Anxiety Sensitivity Index Manual (2nd edition). Worthington, $\mathrm{OH}$ : International Diagnostic Systems

Presentada la nueva Estrategia Nacional de Salud Mental (15 de Ocubre, 2015). Infocop. Recuperado de http://www.infocop.es/ view article.asp?id=5869

Primera experiencia en Murcia de integración de la Psicología en Atención Primaria (s.f.) Infocop. Recuperado de http://colegiopsicologos-murcia.org/web/index.php/ imagen-y-difusion-de-la-profesion/563-elmejor-tratamiento-para-un-estres-postraumatico-es-el-psicologico

Roca, M., Gili, M., García-García, M., Salva, J., Vives, M., Campayo, J. G., \& Comas, A. (2009). Prevalence and comorbidity of common mental disorders in primary care. Journal of Affective Disorders, 119, 52-58.

Sandín, B., Chorot, P., Valiente, R. M., Santed, M. A., \& Lostao, L. (2004). Dimensiones de la sensibilidad a la ansiedad: evidencia confirmatoria de la estructura jerárquica. Revista de Psicopatología y Psicología Clínica, 9, 1933.

Sicras, A., Rejas, J., Navarro R., Serrat, J., Blanca, M., \& Díaz, S. (2007b). Costes y patrón de uso de servicios en pacientes que demandan atención por problemas mentales en asistencia primaria. Gaceta Sanitaria 21(4), 306-319.

Spitzer, R. L., Kroenke, K., Williams, J. B., \& Patient Health Questionnaire Primary Care Study Group. (1999). Validation and utility of a self-report version of PRIME-MD: The PHQ primary care study. JAMA, 282(18), 1737-1744.

Téllez, J. M. (2005). Actualización en Psicofarmacología. Atención Primaria, 36, 61-79

Turpin, Richards, Hope, \& Duffy, (2008). Mejorando el acceso a las terapias psicológicas: una iniciativa nacional para asegurar la prestación de terapias de alta calidad basadas en la evidencia. Papeles del Psicólogo, 29(3), 271-280.

Vermani, M., Marcus, M., \& Katzman, M. A. (2011). Rates of detection of mood and anxiety disorders in primary care: A descriptive, cross-sectional study. Primary Care Companion for CNS Disordors, 13(2), e1-e10.

WHO, \& WONCA (2008). Integrating mental health into primary care: A global perspective. Recuperado de www.who.int/mental health/policy/Integratingmhintoprimarycare2008 lastversion.pdf 